\title{
To manualize, or not to manualize: Is that still the question? A systematic review of empirical evidence for manual superiority in psychological treatment
}

\author{
Femke Truijens $^{1}{ }^{\oplus} \mid$ Levin Zühlke-van Hulzen ${ }^{2} \mid$ Stijn Vanheule ${ }^{1}{ }^{\circledR}$
}

\footnotetext{
${ }^{1}$ Department of Psychoanalysis and Clinical Consulting, Faculty of Psychology and Educational Sciences, Ghent University, Ghent, Belgium

${ }^{2}$ Department of Social Psychology, Faculty of Behavioral and Social Sciences, University of Amsterdam, Amsterdam, The Netherlands

\section{Correspondence}

Femke Truijens, Department of

Psychoanalysis and Clinical Consulting, Faculty of Psychology and Educational Sciences, Ghent University, Henri Dunantlaan 2, B-9000 Ghent, Belgium.

Email: Femke.Truijens@UGent.be
}

\begin{abstract}
Objective: Institutional promotion of psychotherapy manuals as a requirement for evidence-based treatments (EBTs) yields the assumption that manualized treatment is more effective than nonmanualized treatment. This systematic review examines empirical evidence for this claim.

Methods: An electronic database search identified studies that directly or indirectly compared manual-based and nonmanual-based treatment.

Results: Six studies directly compared manualized and nonmanualized treatment (Hypothesis 1). None support manual superiority. Eight meta-analyses indirectly assessed effect sizes of manual-based treatment and control groups (Hypothesis 2). Three support manual superiority, five do not. One meta-analysis and 15 further studies addressed manual adherence as an indirect indicator of manual efficacy (Hypothesis 3). The meta-analysis concluded that manual adherence does not affect outcome, additional studies provided inconclusive results.
\end{abstract}

Conclusions: Manualized treatment is not empirically supported as more effective than nonmanualized treatment. While manual-based treatment may be attractive as a research tool, it should not be promoted as being superior to nonmanualized psychotherapy for clinical practice. 


\section{KEYWORDS}

empirically supported treatment, evidence-based treatment, manual-based treatment, manualization, psychotherapy, treatment efficacy

\section{1 | INTRODUCTION}

Psychotherapy manuals have evolved as a way to secure the application of effective psychotherapies. In 2015 , the American Psychological Association (APA) recognized over 350 evidence-based psychotherapies (EBTs) that are specified by means of treatment manuals (Kazdin, 2015; US Department of Health \& Human Services, 2015). According to Marshall (2009, p. 110) "manuals are intended to direct the application of treatment by specifying: (1) a clear theoretical basis; (2) the number and sequencing of treatment sessions; (3) the content and objectives of each session; and (d) the procedures required to achieve the objective of each session." Manualized treatment increasingly impacts clinical practice and mental health care policy, as influential institutions such as the APA and the National Institute for Health and Care Excellence (NICE) stimulate the dissemination of manual-based EBTs (Norcross, Levant, \& Beutler, 2005). For example, in the UK, the NICE guidelines require that "psychological and psychosocial interventions should be based on the relevant treatment manual(s), which should guide the structure and duration of the intervention" (National Institute for Health and Care Excellence, 2009). This requirement captures the assumption that it is more effective to apply manualized treatment than to provide treatment in a less or nonmanualized form. As this assumption seems vital to justify the dissemination of manual-based EBTs to clinical practice, in this paper, we review the empirical evidence for this assumption.

In clinical practice, however, the use of manuals is heavily critiqued. First, clinicians have argued that a standardized approach might render it impossible to act on the individual characteristics of different clients (Garfield, 1996). While some studies indeed point to difficulties in adapting manual-based treatments to the needs of individual clients (see Hucker \& McCabe, 2012; Marshall, 2009), other research shows that an appropriate amount of attention to the individual client is possible (Jones \& McCabe, 2011). Indeed, increasing attention is given to the "flexibility within fidelity principle," which addresses individualization within manual training (Hamilton, Kendall, Gosch, Furr, \& Sood, 2008). Second, clinicians have argued that manualized treatments only specify treatment for one specific disorder, while clients in practice generally show high levels of comorbidity (Addis, Wade, \& Hatgis, 1999). However, several researchers have shown that manualized treatments are effective towards comorbid disorders besides the target disorder (Kazdin, 2015; Wilson, 1997). A third critique addresses the impossibility for clinicians to gain proficiency in all the different manuals for the extensive array of psychological diagnoses (Addis, 1997). This objection gave rise to the development of transdiagnostic treatment manuals that extrapolate the underlying, shared characteristics of a certain therapeutic approach to different disorders (Kazdin, 2015). These critiques, as well as their proposed solutions, have in common the fact that they focus on problems in the dissemination of manuals from scientific research into clinical practice. That is, the problems concern how manuals could be applied in clinical practice.

However, the question if it is best to apply manuals in clinical practice remains unanswered: Does the use of manuals actually increase therapy effectiveness? And should manuals, therefore, be embraced in clinical practice and training? Although institutes such as NIMH and APA stimulate the use of manuals in clinical practice, the APA also states that there is little research on the effects of the use of manuals in clinical practice (APA 2001, in Chambless \& Ollendick, 2001), yet they suggest that research does not point to decreased effectiveness (see also Addis, Cardemil, Duncan, \& Miller, 2006; Eifert, Schulte, Zvolensky, Lejuez, \& Lau, 1997). The value of manual use was critically addressed in a special issue of Clinical Psychology in 1998 (Treatment Manuals in Clinical Practice, 1998). However, the concerned papers were predominantly based on conceptual or clinical argumentation. Given the current requirement of manuals as the core of evidence-based psychotherapy, it seems crucial to substantiate 
this discussion with empirical evidence. Therefore, in the present article we review the empirical support for EBT's thesis of the superiority of manual-based treatment in psychotherapy.

In a first step, we evaluated the direct evidence for the primary hypothesis that manual-based psychotherapy is more effective than the same kind of psychotherapy applied without a manual. This hypothesis should be satisfied if manualized treatment were indeed superior to nonmanualized treatment in clinical practice. Given the results obtained for the first hypothesis, in the second step, we reviewed the literature for indirect evidence of manual use superiority. For this purpose, we specified two additional hypotheses that should at least be satisfied if the use of manuals were to be favored in the dissemination of EBTs. The second hypothesis therefore purports that if manuals indeed enhance the efficacy of psychotherapy, efficacy studies that use no-treatment, delayed-treatment, minimal treatment or alternative treatment control groups should yield larger efficacy for manual-based therapies than for non-manual-based therapies. If manuals capture the treatment effect then the hypothesized difference should be found regardless of the form of the control group; although it could be larger in the case of intent-to-fail control groups (cf. Westen, Novotny, \& Thompson-Brenner, 2004) than for alternative treatment control groups administered without a treatment manual. For our purposes, therefore, we consider all control groups regardless of their form or credibility. The third hypothesis purports that a low adherence to the treatment manual should be related to lowered treatment effectiveness, while good manual adherence should entail higher effectiveness. This follows from the assumption that a treatment manual captures the specific effective aspects of psychotherapy.

\section{2 | METHODS}

\section{1 | Data collection}

In line with the Moher, Liberati, Tetzlaff, Altman, and The PRISMA Group (2009) statement for systematic reviews, an electronic database literature search was conducted between April and May 2015 and repeated in March 2016. First, a broad search was conducted in the electronic search-engine of the University of Amsterdam (The Netherlands) with the search terms tailor-made and standardized in combination with psychology and psychiatry. Second, a search was conducted in Web of Science with the search terms therapy manual*, treatment manual*, standardized and individualized. These terms were used in all combinations and refined to psychology and psychiatry. As the search term protocol predominantly yielded protocol descriptions in the medical and psychological literature, a search was conducted with the terms nonprotocol*, no protocol*, and unprotocol*. As the few results found by the negation were identical to findings from the previous search, the remainder of the searches was conducted with the manual-related search terms. Third, all the found articles (empirical, theoretical, and review papers) were read and cross-checked for references that were not yet found in the electronic databases (i.e., "the snowball method"). The papers identified in the three searches were used for each of the three hypotheses formulated in the current study.

Additionally, for testing the third hypothesis, a replication was conducted of the literature search done by Webb, DeRubeis, and Barber (2010) on the relation between manual adherence and treatment efficacy. As Webb et al. only reviewed the literature up until April 2009, their search was replicated with identical search terms to identify papers published since then. The replication search was conducted on 17th of March 2016 in the PsycINFO (American Psychological Association, Washington, DC) database.

As the preference for manualized or nonmanualized treatment by research might be prone to allegiance effects, the current project was designed as a joint effort by one social psychologist (research intern) and two clinical psychologists (predoc and tenure), with different clinical and academic experience regarding manualized treatment. The primary literature searches were conducted by the social psychologist, who was not familiar with any clinical opinions on the use of psychotherapy manuals, for avoiding bias and preferences as much as possible in conducting the systematic review. The search process was supervised by the first author, and the findings were assessed, discussed and written up jointly by the three authors. 


\subsection{Data selection}

Papers were included if they satisfied the following conditions: (a) published in English, Dutch, or German language; (b) reporting a direct or indirect comparison of manualized and nonmanualized treatment; and (c) presenting empirical results, either in reports of original data or in reviews or meta-analyses of empirical research. Given the eligibility criterion of empirical results, conceptual, clinical and editorial papers were only used in the snowball search to cross-check any reference to empirical papers. As this review addresses psychotherapeutic manuals, papers were excluded if they covered: (a) pharmacological treatments or treatment of symptoms caused by brain damage; (b) self-help or online treatment without a therapist; (c) manual development.

No restrictions were applied with regard to the year of publication, type of psychotherapy, and type of sample. For transparency, the quality of the included empirical articles was assessed using a Jadad Scale for clinical trials (cf. Jadad et al., 1996). The primary Jadad Scale was complemented with two additional criteria relevant for the hypotheses in this paper. The used criteria were: (a) randomization, (b) double-blinding, (c) description of withdrawals and dropouts, (d) clear inclusion/exclusion criteria, and (e) clear description of interventions. Each article received 1 point per criterion that was both reported and present. Our assessment suffers from what Jadad et al. (1996) mentioned as the "major disadvantage of the instrument described in this paper and of most others" (p. 10) that the assessment is impacted by missing information in the assessed reports. Nonetheless, given the small number of found articles that addressed our research question, all found articles were included in this paper, regardless of their quality.

\section{3 | RESULTS}

\subsection{Search findings}

The final body of literature included six empirical studies that directly assessed the differences in therapy efficacy by manualized or nonmanualized treatment, as well as 16 empirical studies and nine meta-analyses that provided indirect assessments of manual effectiveness. Specifically, our primary hypothesis was covered by six papers, our second additional hypothesis by eight meta-analyses, and our third additional hypothesis by one meta-analysis and 15 empirical studies. Below, we specify the findings per stage of the search process (see Figure 1).

The first search resulted in 36 hits, which were all thoroughly assessed to make sure that all the articles with empirical results were included. Two empirical studies were identified as directly relevant for our primary hypothesis. One review article did not contain empirical information but provided relevant background information in the introduction and discussion and was therefore kept to cross-check references in the third search, see below. The remainder of the findings in the first search consisted of conceptual, theoretical or clinical discussions and clinical research agendas rather than controlled empirical studies, and were therefore excluded from our corpus of empirical literature. The second search resulted in 218 papers, of which two were

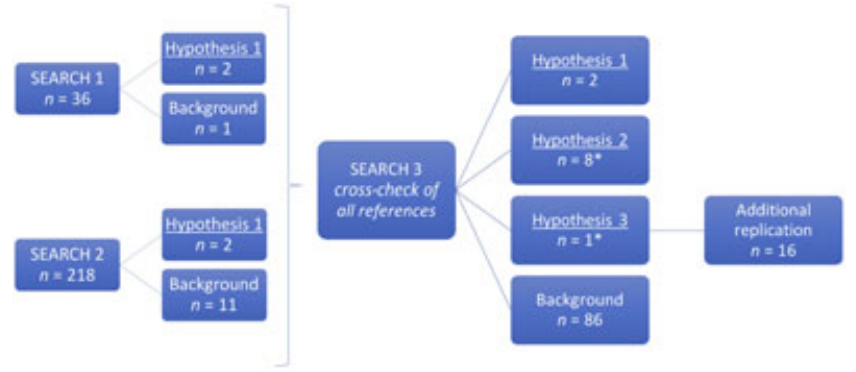

FIGURE 1 Flow chart of findings in three searches and an additional search. ${ }^{*}=$ Meta-analysis [Color figure can be viewed at wileyonlinelibrary.com] 
empirical studies that assessed our primary hypothesis. Furthermore, 11 review articles containing the relevant background information were identified and were kept to cross-check references in the third search, see below. The remainder of the findings in the second search again yielded conceptual, theoretical or clinical discussions rather than empirical research and were therefore excluded from our corpus of empirical literature. The third search resulted in the identification of two empirical studies that covered our primary hypothesis, eight meta-analyses that covered our second additional hypothesis, and one meta-analysis that contained empirical findings concerning our third additional hypothesis. Another 86 review articles were identified based on a keyword hit in the abstract, but none of these contained empirical information regarding our hypotheses. Additionally, the meta-analysis that was found to cover our third hypothesis was replicated. This replication search resulted in 15 empirical studies that were published after the meta-analysis. Full reference lists of all included and excluded papers are available upon request. In the following section, we present the results of the literature review ordered per hypothesis.

Hypothesis 1 Is manual-based psychotherapy more effective than the same kind of psychotherapy applied without a manual?

In the first step, we examined the hypothesized superiority of manual-based psychotherapy over the same kind of psychotherapy applied without a manual. Only six studies were identified that directly assessed this primary hypothesis. One study showed manual use superiority. Three studies demonstrated that the efficacy of manualized and nonmanualized treatments did not differ. Two studies observed that nonmanualized psychotherapy was more effective. Table 1 shows the quality assessment by means of the Jadad Scale (cf. Jadad et al., 1996) and conclusions about manual superiority.

Schulte, Künzel, Pepping, and Schulte-Bahrenberg (1992) tested the effectiveness of a manualized and a nonmanualized cognitive-behavioral therapy (CBT) for phobias. The nonmanualized treatment was planned by the therapist based on a functional analysis of the problems of the individual client (Schulte, 1981), using a nonspecified amount and order of cognitive-behavioral techniques. In the manualized condition, the order and type of cognitivebehavioral techniques was specified a priori and without preliminary information on the nature of the client's phobic anxiety complaints. The manualized treatment was found to be more effective. The difference was explained by the exceptional effectiveness of "direct exposure" for the treatment of phobias: Nonmanualized treatment that included direct exposure appeared to be as effective as the manualized treatment that included direct exposure. In other words, when therapists were as prone to use this technique in nonmanualized treatment as they would be in manualized treatment, the difference in effect would nullify. This suggests the utility of a manual as an impetus for therapists for using a specific technique. Translated to evidence for manual use, however, this suggests a moderating role of the manual to disseminate specific effective interventions at most, but it does not yield support for the efficacy of manualized treatment per se.

Van Hout, Emmelkamp, Koopmans, Bögels, and Bouman (1994) conducted a similar study on CBT for obsessivecompulsive disorders. They did not find a difference in effectiveness between the manualized and the nonmanualized treatment. In his/her doctoral research, Fane (1998) did not find differences between manual-based group therapy for sexual offenders and nonmanualized group therapy with regard to aggression and attitude management. As the report was not published, no information on the quality of the assessment was available, hence quality assessment was not applicable. Morgenstern, Blanchard, Morgan, Labouvie and Hayaki (2001) compared the effectiveness of a manualized CBT to a nonmanualized CBT and treatment-as-usual (TAU) for addiction. In the TAU condition, therapists were instructed to provide therapy as they would to their regular clients but to use no CBT techniques. Therapists in all conditions received comparable supervision. No efficacy differences were found for either one of the therapies.

Ghaderi (2006) showed that for bulimia nervosa both a manualized version of CBT and a nonmanualized version are effective although the nonmanualized version showed a lower level of nonresponding patients and a lower level of relapse. The nonmanualized therapy was based on continuous logical functional analysis, the manualized therapy followed the 


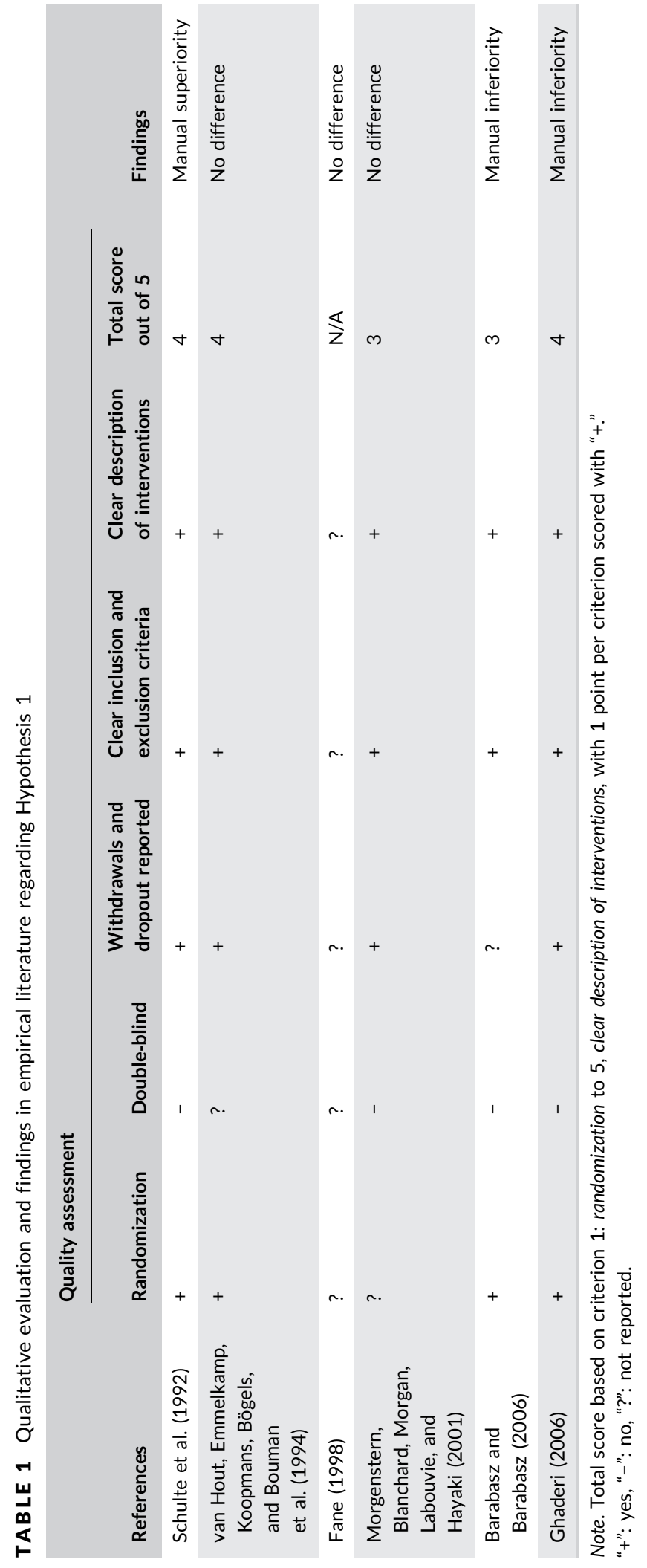


manual of Fairburn, Marcus, and Wilson (1993). In the same year Barabasz and Barabasz (2006) investigated hypnotherapy for complicated irritable bowel syndrome. They found that manualized hypnotic inductions were less effective than tailored hypnotic inductions, although patients in both conditions showed improvement.

The six studies that conducted a direct comparison of manualized and nonmanualized psychotherapy do not support the hypothesized superiority of manual-based psychotherapy. On the contrary, three studies found no substantial difference, two observed the opposite, and the single study that supports superiority starts from a single specific intervention that appeared to be exceptionally effective, regardless of the administration via a manual. If we control for quality and exclude studies with a score of three out of five possible points or lower, still, one inconclusive, one negative, and one indifferent result remain. This suggests that there is no sound evidence for the superiority of manualized therapy. Yet, the limited amount of rigorous studies prevents from drawing firm conclusions on this vital hypothesis on evidencebased treatment. Given the lack of support for the direct hypothesis of manual superiority, we formulated two additional hypotheses that may support manual superiority indirectly.

Hypothesis 2 Are effect sizes of manual-based therapies larger for manual-based than for nonmanualized treatments?

Secondly, we evaluated whether in psychotherapy efficacy studies that use no-treatment, delayed-treatment, minimal treatment or alternative treatment control groups, larger effect sizes can be observed for manual-based therapies than for non-manual-based therapies. To measure psychotherapy efficacy, researchers usually calculate the difference between pretreatment and posttreatment symptom scores in an experimental/treatment condition and a control condition, often using effect sizes to indicate the strength of the found differences (Olejnik \& Algina, 2000). Eight meta-analyses addressed the average effect size of manualized and nonmanualized psychotherapy efficiency studies. Three of these concluded that manual-based therapies were superior, but four found no difference, and one observed the opposite. Table 2 gives an overview of the types of control conditions that were included in each meta-analysis as well as their findings towards manual superiority.

A meta-analysis on the efficiency of short-term psychodynamic treatments (Anderson \& Lambert, 1995) found that studies using manuals showed a higher effect size than studies of nonmanualized treatments on follow-up measurements

TABLE 2 Features and findings in the empirical literature regarding Hypothesis 2

\begin{tabular}{|c|c|c|c|}
\hline & $\begin{array}{l}\text { No-treatment, delayed treatment } \\
\text { and/or minimal treatment control } \\
\text { group included }\end{array}$ & $\begin{array}{l}\text { Alternative treatment } \\
\text { control group included }\end{array}$ & Finding \\
\hline $\begin{array}{l}\text { Anderson and Lambert } \\
(1995)^{\mathrm{a}}\end{array}$ & Yes & Yes & Manual superiority \\
\hline $\begin{array}{l}\text { DiGiuseppe and Tafrate } \\
(2003)^{a}\end{array}$ & Yes & Yes & Manual superiority \\
\hline $\begin{array}{l}\text { Stewart and Chambless } \\
(2009)^{\mathrm{a}}\end{array}$ & Yes & No & Manual superiority \\
\hline Robinson et al. (1990) ${ }^{a}$ & Yes & Yes & No difference \\
\hline Shadish and Baldwin (2005) & Yes & Not specified & No difference \\
\hline $\begin{array}{l}\text { Shadish, Navarro, Matt, and } \\
\text { Philipps (2000) }\end{array}$ & Yes & No & No difference \\
\hline Saini $(2009)^{a}$ & Yes & Yes & No difference \\
\hline Wright et al. (2007) ${ }^{a}$ & Yes & No & Manual inferiority \\
\hline
\end{tabular}

a Meta-analysis. 
(if specified, follow-up measures were administered either within or after 6 months posttreatment). Yet, the difference immediately after the intervention was not statistically significant. In a meta-analysis on treatments for anger, DiGiuseppe and Tafrate (2003) considered manual usage as a covariate and observed that using a manual yielded significantly higher effect sizes. Stewart and Chambless (2009) also found larger effect sizes in case of manual-use.

Robinson, Berman, and Neimeyer (1990) did a meta-analysis of efficiency studies of psychotherapies for depression. They found no difference in effect size between manual-based and nonmanualized treatments. Shadish, Navarro, Matt and Phillips (2000) developed criteria to score the clinical representativeness of psychotherapy efficiency studies and conducted a meta-analysis to examine if these criteria were related to effect size. One of the criteria that they used was the use of a therapy manual. The analysis showed that in a highly clinically representative sample of psychotherapy efficiency studies and also in a randomly selected sample there was no association between effect size and the use of a treatment manual. More recent studies using the same coding system found comparable results (Shadish \& Baldwin, 2005). In a meta-analysis on treatments for anger, Saini (2009) also observed that no statistically significant difference in effect size could be observed between manualbased and non-manual-based therapies. Finally, one study observed a strong negative association between effect size and the use of therapy manuals (Wright, Sabourin, Mondor, McDuff, \& Mamodhoussen, 2007).

As the Jadad Scale is designed for trials, quality could not formally be scored for the identified meta-analyses. Nonetheless, we checked whether the meta-analyses took the criteria of our Jadad Scale into account in the selection of their corpus of literature. Randomization was used as a criterion in four out of eight meta-analyses (Saini, 2009; Shadish \& Baldwin, 2005; Shadish, Navarro, Matt \& Phillips, 2000; Stewart \& Chambless, 2009). Wright et al. (2007) mentioned randomization but did not use it as an eligibility criterion. Wright et al. (2007) did select studies based on the clarity of their interventions. Furthermore, none of the papers reported whether randomization, double-blind assessment, withdrawals or dropouts, inclusion or exclusion criteria or description of interventions were described in the studies incorporated in the meta-analyses. Therefore, as the study quality has not been evaluated systematically, the results of these meta-analyses should be interpreted with care.

In sum, three meta-analyses of the average effect size of psychotherapy efficacy studies show an advantage for studies employing manuals, one shows a disadvantage and four find no relation between manual use and effect size. These results are heterogeneous because of the varying nature of the control conditions that were entertained in the reviewed studies. A number of meta-analyses included alternative treatment control groups besides no-treatment, delayed treatment or minimal treatment control groups (see Table 2). As the comparison between manualization and nonmanualization is less straightforward in the case of alternative treatment, inclusion of alternative treatment control groups might limit the discriminative power to demonstrate the hypothesized differences. If we only consider the meta-analyses that compared manualized treatment to no-treatment, delayed treatment or nonmanualized minimal treatment control groups, the results remain inconclusive, as one finds manual use superiority, one finds manual use inferiority and one finds no difference. Taken together, the reviewed studies don't provide sound support for the hypothesis that manualized psychotherapies yield larger effect sizes than nonmanualized psychotherapies.

\section{Hypothesis 3 Is manual adherence indicative of effectiveness of treatment?}

Third, we studied the hypothesis that low adherence to a treatment manual is related to lowered treatment effectiveness. We identified one meta-analysis that investigated the association between treatment adherence and treatment efficacy in psychotherapy (Webb et al., 2010). The meta-analysis included 32 studies that either (a) investigated individual psychotherapy; (b) included a quantifiable measure of both adherence and treatment outcome assessed no later than 6 months after treatment termination; (c) assessed adherence based on videotaped, audiotaped, or transcribed therapy sessions rated by experts or trained raters; (d) included a clinical population; (e) comprised at least five patients in each of the treatment groups; and (f) were published in English. Analysis showed that there was no association between adherence and treatment efficiency in the overall sample or for any specific disorder. 

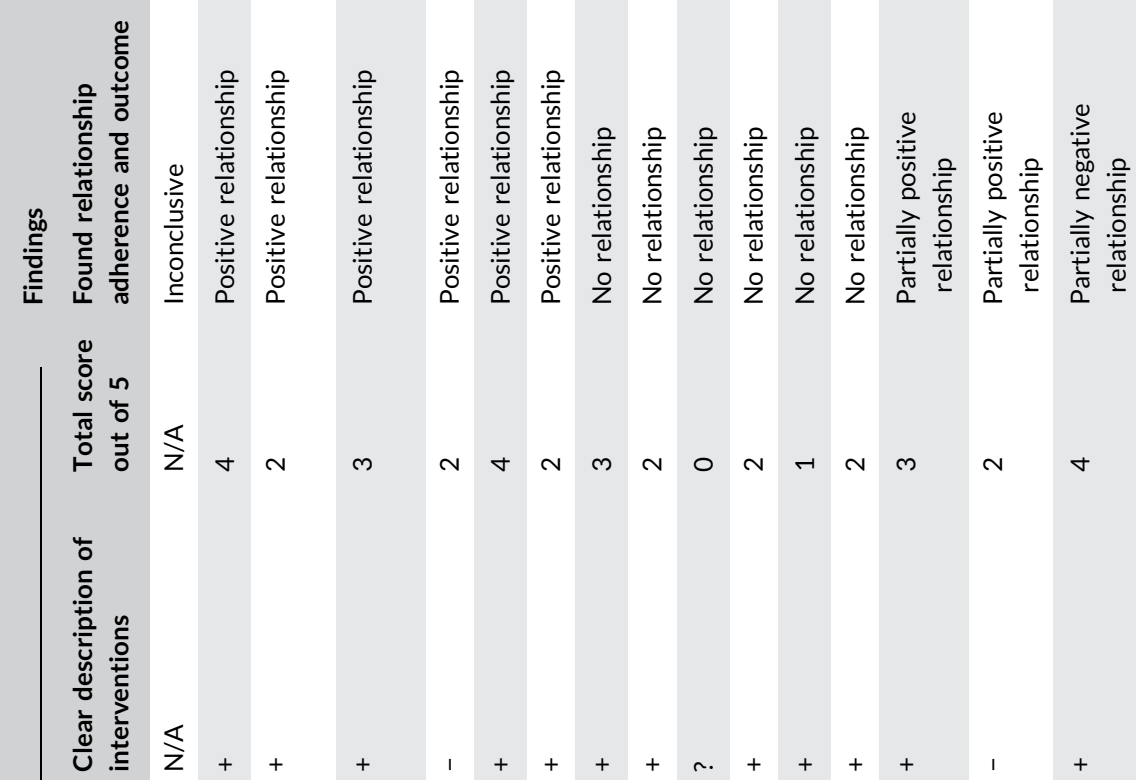

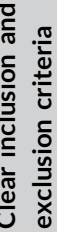

$\frac{5}{z}$

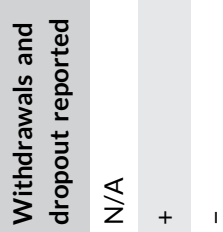

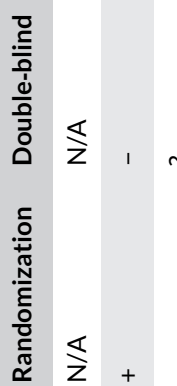

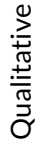

产

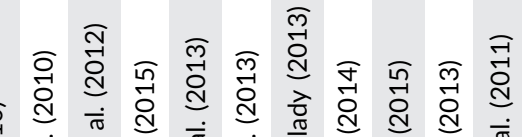

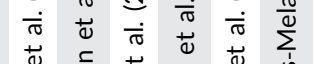

मं $\dot{\sigma} \quad \dot{\sigma}$

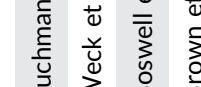

है

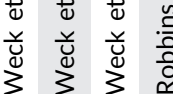

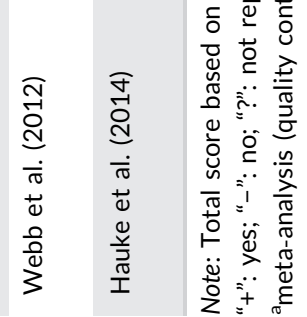


Additionally, we replicated the search by Webb et al. (2010) to identify papers that were published since the time of their review. Sixteen papers were found by using the same search term and inclusion criteria as were used by Webb et al. (2010), of which one was excluded (Chapman \& Schoenwald, 2011) as the article was based on the same data analysis as a previous study (Schoenwald, Sheidow, \& Chapman, 2009). Table 3 shows the quality assessment by means of the Jadad Scale (cf. Jadad et al., 1996) and conclusions about manual superiority.

Five of the studies found a positive association of treatment adherence and efficiency (Haug et al., 2016; Schoenwald et al., 2009; Sexton \& Turner, 2010; Strunk, Brotman, \& DeRubeis, 2010; Suchman, Decoste, Rosenberger, \& McMahon, 2012) and six found no relation between treatment adherence and efficiency (Boswell et al., 2013; Brown et al., 2013; Campos-Melady, 2013, Weck et al., 2013; Weck, Grikscheit, Höfling, \& Stangier, 2014; Weck, Richtberg, Jakob, Neng, \& Höfling, 2015). One study found a positive association between treatment adherence and efficiency in one sample and no relation in a second sample (Webb et al., 2012). One study found a positive association between treatment adherence and efficiency for some but not all outcome measures (Robbins et al., 2011). One study found a negative association between treatment adherence and some but not all outcome measures (Hauke et al., 2014). Weck, Grikscheit, Jakob, Höfling, and Stangier (2015) compared only treatment outcomes for participants that were classified as treatment failure or clear treatment, pertaining to $37 \%$ of all participants that were treated. In this selective sample they found a positive association between treatment adherence and outcome.

In sum, before April 2009, 32 studies addressed the relation between manual adherence and treatment efficiency, and hitherto another 15 studies were published on the topic. Given the ambiguity and the often insufficient quality in the corpus of recent studies, the findings remain as inconclusive as the body of literature analyzed by Webb et al. (2010). Therefore, their conclusion that better manual adherence does not automatically entail higher treatment effectiveness was not substantially challenged by recent empirical literature. Although this leaves the question of an association between adherence and treatment efficiency open for discussion, the findings are insufficient as proof of indirect evidence for manual use superiority.

\section{DISCUSSION}

In the paradigm of evidence-based treatment, manual-based treatments are increasingly implemented both in clinical practice and training to secure the dissemination of empirically supported psychotherapies. This rests on the assumption that manual-based treatment is more effective than nonmanualized treatment. In this paper, we systematically reviewed the available empirical evidence for this basic assumption based on the primary hypothesis that (a) manual-based psychotherapy is more effective than the same kind of psychotherapy applied without a manual; and two additional indirect hypotheses that (b) in no-treatment, delayed-treatment, minimal treatment or alternative treatment control groups, manual-based therapies yield larger effect sizes than non-manual-based therapies, and that (c) good manual adherence entails higher effectiveness. A notably small body of literature was found that directly or indirectly addressed the efficacy of manualization.

The primary hypothesis of the superiority of manualized treatment over nonmanualized treatment was not supported by the available empirical literature. For the first hypothesis, only six studies were identified that directly assessed the primary assumption of manual superiority. Out of these six, four studies showed no difference between manualized and nonmanualized treatment, and two studies proved the opposite, indicating that nonmanualized psychotherapy is actually more effective. The only study that found manualized treatment to be superior was explained by the effect of a specific exposure intervention (Schulte et al., 1992). This example showcases the simple logic that it is more effective to offer an effective intervention than not to offer the same technique. As exposure techniques often meet with reluctance from therapists, which prohibits patients from optimally benefiting from the intervention (cf. Powers \& Deacon, 2013), these findings underscore the fact that therapists should be informed about scientific evidence, and hence that treatment manuals may be utilized as 
an impetus to disseminate effective techniques (cf. Wampold \& Imel, 2015, for a discussion). However, this utility still does not support the assumption that manualized treatment is more effective than nonmanualized treatment per se.

Given the limited and inconclusive empirical support for the primary hypothesis in evidence-based treatment, in the second step, we reviewed the literature for indirect evidence of manual superiority. For this purpose, we specified two additional hypotheses that should at least be satisfied if the use of manuals were to be favored in the dissemination of EBTs. Specifically, empirical support would be needed to propose the manual as a moderator for treatment efficacy. Concerning the second hypothesis, three meta-analyses indicated that manual-based therapies were superior, four found no difference, and one observed that nonmanualized psychotherapies actually yielded larger effect sizes. A correction for quality of the included empirical studies would not leave out much literature. Here, we have to remark that it is fairly complex to meaningfully compare effect sizes of treatments that are so different in nature, given their varied understanding and operationalization of treatment, control groups, diagnosis, and outcome. First and foremost, this underlines how the universal hypothesis of manual efficacy is in trouble with respect to empirical support, both as a direct and as a moderating factor.

With respect to the third hypothesis, one meta-analysis concluded that manual adherence does not affect outcome, and 15 additional studies provided inconclusive results. If manuals themselves were to have an impact on treatment efficacy, then, at least a less strict application of manuals should yield a lowered treatment outcome. In line with the conclusion of Webb et al. (2010), we did not find sound evidence for this indirect hypothesis of manual superiority. Of the 15 studies that were conducted after the meta-analysis of Webb et al. (2010), five found a positive relationship between adherence and treatment efficiency. These should be taken into consideration despite the contrasting results with the remainder of the corpus, as it is more common to assume that there is no association while there could be one (i.e. type II error) than to assume an association while there is none (i.e., type I error; cf. Mook \& Parker, 2001). As such, the suggestion that adherence and fidelity to treatment principles may impact a positive treatment outcome remains a worthwhile avenue for further research. However, as an indicator for efficacy of the manual as a general principle for clinical practice, this conflicting body of evidence is insufficient. Moreover, it challenges the proposed moderating role of manuals towards treatment effect. With regard to clinical practice, this finding does not necessarily dispute the utility of manuals for training and dissemination of effective interventions, yet it does refute the a priori requirement of manuals for psychological treatment.

Overall, this review shows that the hypothesized superiority of manual-use in psychotherapy cannot be established, which points to a severe problem in the justification of EBT dissemination. The current study was set up to review the direct evidence for a crucial basic assumption that is embedded in general clinical recommendations. Given the small number of studies that directly assessed the primary hypothesis of manualbased psychotherapy, we also gathered evidence for two additional indirect hypotheses. None of these hypotheses yielded substantial evidence nor even a sound and systematic consideration of the superiority of manual use. Based on these limited and inconclusive findings, it might be argued that clinical practice is becoming influenced by a standardized approach that does not live up to its own claim of superiority in comparison to regular psychotherapies in which clinicians take individual and non-controllable decisions for each separate client.

In interpreting this literature review, a number of limitations have to be taken into account. First, given the indirect nature of the additional hypotheses, the search was notably heterogeneous. This is due to the remarkably small number of studies that focused on the primary hypothesis, which urged us to add two indirect hypotheses. It is striking that only six studies - of which a substantial part was published before the rise in popularity of EBTdirectly entertained the most basic assumption of EBT, notwithstanding the 350 manual-based treatments that are recognized as EBT today (cf. Kazdin, 2015). Therefore, this limitation may be taken as exemplary of a vital limitation in the evidence-base of EBT in psychotherapy. Second, given the limited number of articles found that addressed our research questions directly, we did not find further leads to identify additional or unpublished data. We would expect, however, that if such data were available, they would surely be published. Third, the current study does not yield a meta-analysis of the found empirical evidence. As the primary hypothesis was covered by only six studies 
that either did not indicate a difference or favored nonmanualized over manualized treatment, a meta-analysis would not have added to the conclusion of lacking evidence of manual-based treatment. Moreover, as the indirect hypotheses did not contain a sound or similar means of assessing manual superiority, conducting a meta-analysis on indirect and methodologically divergent studies would have been methodologically questionable as well as undermining the signalization of the fundamental lack of empirical interest in the primary assumption. Finally, in this study, we did not distinguish between evidence for specific disorders. As the APA, NICE and other (inter) national institutions stimulate manual-based treatment as a basic principle in treatment guidelines, we treated the evidence likewise. However, specification of the evidence for disorders, symptom severity and chronicity, and specific patient populations, could be utilized in future research to specify the feasibility of manual-based treatment for specific clinical cases. Based on this review, we are not inclined to call for more research to settle the dispute about manualization in general; rather, we urge both researchers and clinicians to go beyond the dichotomy, as the next step in understanding what works for whom in psychotherapy.

It is crucial to note that, in the first place, psychotherapy manuals are not developed as practice guidelines but as tools for standardization in clinical research (Vanheule, 2009). In the EBT paradigm, a form of psychotherapy can be established as evidence-based if its efficacy has been shown in at least two independent randomized controlled trials or similarly controlled and systematic research designs (Chambless \& Hollon, 1998). The logic behind this study design is called "interventionist causality" (Kendler \& Campbell, 2009): If an experimental and a control group are identical except for the intervention, then a difference in the "outcome" must be due to the intervention. With this logic, it is crucial that the intervention is identical for every participant in the experimental group; otherwise, the found differences could be due to arbitrary sample differences instead of the intervention itself (Woodward, 2003). Therefore, the function of psychotherapy manuals is to standardize the intervention such that causal statements on the efficacy of psychotherapy are enabled (cf. Woodward, 2003). Consequently, evidence is systematically gathered for manualized treatments, which does not imply that manual-based treatment is more effective but merely that manualized treatment is methodologically favored.

Indeed, if an outcome study supports the efficacy of a manualized therapy, this does not support the use of the manual itself but it implies that certain "ingredients" of the intervention that were specified in the manual work. Illustrative is the study of Schulte et al. (1992), in which the advantage found for manual-based cognitive-behavioral treatment for phobias appeared to be due to the exceptional effectiveness of a specific therapeutic technique rather than due to the application of the manual per se. This example shows that whether a therapy should be manualized or nonmanualized is not the right question to ask. Far more important is to understand what it is that works within a specific treatment approach and for whom (cf. Wampold \& Imel, 2015). Moreover, it is not necessary that "what works" is limited to one specific ingredient-such as the specific exposure for phobias-that works for each demarcated diagnosis.

This thesis is central to a promising line of inquiry pioneered by Chorpita, Daleiden, and Weisz (2005a, 2005b) among others (cf. Norcross, Levant, \& Beutler, 2005). This line of research focuses on individualized manualization, in which an experienced therapist can select different manual-based modules that can be custom-fit to the needs, complaints and preferences of individual patients. This could furthermore allow for a transdiagnostic approach of comorbid symptomatology, as it moves away from a pre-set diagnosis-based treatment plan and towards therapy based on core processes in individually experienced and possibly overlapping complaints (Kazdin, 2015). Importantly, this line of research does not provide universal support for efficacy of "the manual" per se but it does promote manualized interventions as tools within an interpersonal therapeutic context. This is in line with the clinical integration of theory, scientific evidence and clinical experience that is being increasingly promoted as evidence-based practice (cf. APA Presidential Task Force on Evidence-Based Practice, 2006), and suggests the value of individualization beyond a more general promotion of a one-size-fits-all manualization.

The current lack of empirical support for the assumed superiority of manuals as a universal principle for clinical practice urges researchers to rethink the function of their evidence. Instead of taking the evidence for using an effective treatment manual as a conclusion, it should be taken as a starting point to investigate which components of the therapy, 
and which steps in the therapy process make the manual work. In this, the current general dichotomy between manualized and nonmanualized treatment is not fruitful. Indeed, it arrests researchers' and stakeholders' interest at the level of the manual and at the level of the sample, which is neither an epistemically nor clinically fruitful imperative (cf. Desmet, 2013; Truijens, 2016). Epistemic progress seems to require that the researcher does not embrace manual-use a priori and generally but carefully scrutinizes when, why and how specific techniques can be utilized in clinical practice.

Importantly, to get beyond the dichotomy, it is similarly crucial for clinicians to not reject manuals a priori, as that would merely be a rejection of form. Yet the content of treatments could be improved if clinicians try specific techniques and treatment plans. This asks for going beyond the manual/non-manual dichotomy but just as well beyond the divide between researchers and clinicians. Only a joint effort between researchers and clinicians will provide both epistemic and clinical progression. Moreover, to elucidate processes that explain therapeutic success or failure it seems crucial to study specific (series of) cases that allow for a close look at clinical situations, focusing on how specific interventionsmanualized or nonmanualized-foster or hinder change. Along this way, the ethos of EBT - disseminating research findings to clinical practice - might be established much better than by promoting manuals as such.

\section{ACKNOWLEDGEMENTS}

The authors thank Dr. Alvin Westmaas (University of Amsterdam) for providing internship supervision, Drs Rosa De Geest (Ghent University) for collaboration at the conference and the Department of Psychoanalysis and Clinical Consulting (Ghent University) for feedback and support throughout the process.

\section{ORCID}

Femke Truijens (D) http://orcid.org/0000-0003-0790-7003

Stijn Vanheule (D) http://orcid.org/0000-0001-8580-5809

\section{REFERENCES}

Addis, M. E. (1997). Evaluating the treatment manual as a means of disseminating empirically validated psychotherapies. Clinical Psychology: Science and Practice, 4, 1-11.

Addis, M. E., Cardemil, E. V., Duncan, B. L., \& Miller, S. D. (2006). Does manualization improve therapy outcomes? In J. C. Norcross, L. E. Beutler, \& R. F. Levant (Eds.), Evidence-based practices in mental health: Debate and dialogue on the fundamental questions (pp. 131-160). Washington, DC, US: American Psychological Association.

Addis, M. E., Wade, W. A., \& Hatgis, C. (1999). Barriers to dissemination of evidence-based practices: Addressing practitioners' concerns about manual-based psychotherapies. Clinical Psychology: Science and Practice, 6, 430-441.

Anderson, E. M., \& Lambert, M. J. (1995). Short-term dynamically oriented psychotherapy: A review and meta-analysis. Clinical Psychology Review, 15, 503-514.

APA Presidential Task Force on Evidence-Based Practice (2006). Evidence-based practice in psychology. American Psychologist, 61, 271-285.

Barabasz, A., \& Barabasz, M. (2006). Effects of tailored and manualized hypnotic inductions for complicated irritable bowel syndrome patients. International Journal of Clinical and Experimental Hypnosis, 54, 100-112.

Boswell, J. F., Gallagher, M. W., Sauer-Zavala, S. E., Bullis, J., Gorman, J. M., Shear, M. K., ... Barlow, D. H. (2013). Patient characteristics and variability in adherence and competence in cognitive-behavioral therapy for panic disorder. Journal of Consulting and Clinical Psychology, 81, 443-454.

Brown, L. A., Craske, M. G., Glenn, D. E., Stein, M. B., Sullivan, G., Sherbourne, C., ... Rose, R. D. (2013). CBT competence in novice therapists improves anxiety outcomes. Depression and Anxiety, 30, 97-115.

Campos-Melady, M. (2013). Therapist adherence and competence as predictors of client outcomes in adolescent substance use treatment (Unpublished Doctoral dissertation). University of New Mexico, USA.

Chambless, D. L., \& Hollon, S. D. (1998). Defining empirically supported therapies. Journal of Consulting and Clinical Psychology, 66, 7-18.

Chambless, D. L., \& Ollendick, T. H. (2001). Empirically supported psychological interventions: Controversies and evidence. Annual Review of Psychology, 52, 685-716. 
Chapman, J. E., \& Schoenwald, S. K. (2011). Ethnic similarity, therapist adherence, and long-term multisystemic therapy outcomes. Journal of Emotional and Behavioral Disorders, 19, 3-16.

Chorpita, B. C., Daleiden, E., \& Weisz, J. R. (2005b). Modularity in the design and application of therapeutic interventions. Applied \& Preventive Psychology, 11, 141-156.

Chorpita, B. F., Daleiden, E. L., \& Weisz, J. R. (2005a). Identifying and selecting the common elements of evidence based interventions: A distillation and matching model. Mental Health Services Research, 7, 5-20.

Desmet, M. (2013). Experimental versus naturalistic psychotherapy research: Consequences for researchers, clinicians, policy makers and patients. Psychoanalytische Perspectieven, 31, 59-78.

DiGiuseppe, R., \& Tafrate, R. C. (2003). Anger treatment for adults: A meta-analytic review. Clinical Psychology: Science and Practice, 10, 70-84.

Eifert, G. H., Schulte, D., Zvolensky, M. J., Lejuez, C. W., \& Lau, A. W. (1997). Manualized behavior therapy: Merits and challenges. Behavior Therapy, 28, 499-509.

Fairburn, C. G., Marcus, M. D., \& Wilson, G. T. (1993). Cognitive behaviour therapy for binge eating and bulimia nervosa: A comprehensive treatment manual. In C. G. Fairburn \& G. T. Wilson (Eds.), Binge Eating: Nature, Assessment and Treatment (pp. 361-404). New York: Guilford Press.

Fane, R. B. (1998). The effect of structured and unstructured group therapy on the anger and attitudes of domestic violence perpetrators (Unpublished doctoral dissertation). University of Louisville, Kentucky, USA.

Garfield, S. L. (1996). Some problems associated with "validated" forms of psychotherapy. Clinical Psychology: Science and Practice, 3, 218-229.

Ghaderi, A. (2006). Does individualization matter? A randomized trial of standardized (focused) versus individualized (broad) cognitive behavior therapy for bulimia nervosa. Behaviour Research and Therapy, 44, 273-288.

Hamilton, J. D., Kendall, P. C., Gosch, E., Furr, J. M., \& Sood, E. (2008). Flexibility within fidelity. Journal of the American Academy of Child \& Adolescent Psychiatry, 47, 987-993.

Haug, T., Nordgreen, T., Öst, L. G., Tangen, T., Kvale, G., Hovland, O. J., ... Havik, O. E. (2016). Working alliance and competence as predictors of outcome in cognitive behavioral therapy for social anxiety and panic disorder in adults. Behaviour Research and Therapy, 77, 40-51.

Hauke, C., Gloster, A. T., Gerlach, A., Richter, J., Kircher, T., Fehm, L., \& Deckert, J. (2014). Standardized treatment manuals: Does adherence matter? Sensoria: A Journal of Mind, Brain \& Culture, 10, 1-13.

van Hout, W. J., Emmelkamp, P. M., Koopmans, P. C., Bögels, S. M., \& Bouman, T. K. (1994). Individualized versus standardized therapy: A comparative evaluation with obsessive-compulsive patients. Clinical Psychology \& Psychotherapy, 1, 95-100.

Hucker, A., \& McCabe, M. P. (2012). Manualized treatment programs for FSD: Research challenges and recommendations. The Journal of Sexual Mmedicine, 9, 350-360.

Jadad, A. R., Moore, R. A., Carroll, D., Jenkinson, C., Reynolds, D. J. M., Gavaghan, D. J., \& McQuay, H. J. (1996). Assessing the quality of reports of randomized clinical trials: Is blinding necessary? Controlled Clinical Trials, 17, 1-12.

Jones, L. M., \& McCabe, M. P. (2011). The effectiveness of an internet-based psychological treatment program for female sexual dysfunction. The Journal of Sexual Medicine, 8, 2781-2792.

Kazdin, A. E. (2015). Treatment as usual and routine care in research and clinical practice. Clinical Psychology Review, 42, 168-178.

Kendler, K. S., \& Campbell, J. (2009). Interventionist causal models in psychiatry: Repositioning the mind-body problem. Psychological Medicine, 39, 881-887.

Marshall, W. L. (2009). Manualization: A blessing or a curse? Journal of Sexual Aggression, 15, 109-120.

Moher, D., Liberati, A., Tetzlaff, J., Altman, D. G., \& The PRISMA Group (2009). Preferred reporting items for systematic reviews and meta-analyses: The PRISMA statement. PLOS Medicine, 6, e1000097. https://doi.org/10.1371/journal.pmed.1000097

Mook, D. G., \& Parker, S. (2001). Psychological research: The ideas behind the methods. New York: Norton.

Morgenstern, J., Blanchard, K. A., Morgan, T. J., Labouvie, E., \& Hayaki, J. (2001). Testing the effectiveness of cognitivebehavioral treatment for substance abuse in a community setting: Within treatment and posttreatment findings. Journal of Consulting and Clinical Psychology, 69, 1007-1017.

National Institute for Health and Care Excellence (2009). Depression in adults: recognition and management. Retrieved from https://www.nice.org.uk/guidance/CG90/chapter/Key-priorities-for-implementation

Norcross, J., Levant, R., \& Beutler, L. (2005). Evidence-based practices in mental health: Debate and dialogue on the fundamental questions. Washington, DC: American Psychological Association Press.

Olejnik, S., \& Algina, J. (2000). Measures of effect size for comparative studies: Applications, interpretations, and limitations. Contemporary Educational Psychology, 25, 241-286.

Powers, M. B., Deacon, B. J. (Eds.) (2013). Dissemination of empirically supported treatments for anxiety disorders [Special Issue]. Journal of Anxiety Disorders, 27.

Robbins, M. S., Feaster, D. J., Horigian, V. E., Puccinelli, M. J., Henderson, C., \& Szapocznik, J. (2011). Therapist adherence in brief strategic family therapy for adolescent drug abusers. Journal of Consulting and Clinical Psychology, 79, 43-53.

Robinson, L. A., Berman, J. S., \& Neimeyer, R. A. (1990). Psychotherapy for the treatment of depression: A comprehensive review of controlled outcome research. Psychological Bulletin, 108, 30-49. 
Saini, M. (2009). A meta-analysis of the psychological treatment of anger: Developing guidelines for evidence-based practice. Journal of the American Academy of Psychiatry and the Law Online, 37, 473-488.

Schoenwald, S. K., Sheidow, A. J., \& Chapman, J. E. (2009). Clinical supervision in treatment transport: Effects on adherence and outcomes. Journal of Consulting and Clinical Psychology, 77, 410-421.

Schulte, D. (1981). Schema für Probleemanalyse und Therapie Planung, (A framework for problem analysis and therapy planning). Unpublished manuscript.

Schulte, D., Künzel, R., Pepping, G., \& Schulte-Bahrenberg, T. (1992). Tailor-made versus standardized therapy of phobic patients. Advances in Behaviour Research and Therapy, 14, 67-92.

Sexton, T., \& Turner, C. W. (2010). The effectiveness of functional family therapy for youth with behavioral problems in a community practice setting. Journal of Family Psychology, 24, 339-348.

Shadish, W. R., \& Baldwin, S. A. (2005). Effects of behavioral marital therapy: A meta-analysis of randomized controlled trials. Journal of Consulting and Clinical Psychology, 73, 6-14.

Shadish, W. R., Navarro, A. M., Matt, G. E., \& Phillips, G. (2000). The effects of psychological therapies under clinically representative conditions: A meta-analysis. Psychological Bulletin, 126, 512-529.

Stewart, R. E., \& Chambless, D. L. (2009). Cognitive-behavioral therapy for adult anxiety disorders in clinical practice: A meta-analysis of effectiveness studies. Journal of Consulting and Clinical Psychology, 77, 595-606.

Strunk, D. R., Brotman, M. A., \& DeRubeis, R. J. (2010). The process of change in cognitive therapy for depression: Predictors of early inter-session symptom gains. Behaviour Research and Therapy, 48, 599-606.

Suchman, N. E., Decoste, C., Rosenberger, P., \& McMahon, T. J. (2012). Attachment-based intervention for substance-using mothers: A preliminary test of the proposed mechanisms of change. Infant Mental Health Journal, 33, 360-371.

Truijens, F. L. (2016). Do the numbers speak for themselves? A critical analysis of procedural objectivity in psychotherapeutic efficacy research. Synthese, 194, 4721-4740.

US Department of Health and Human Services (2015). National registry of evidence-based programs and practices (NREPP). Retrieved from http://nrepp.samhsa.gov/

Vanheule, S. (2009). Psychotherapy and research: A relation that needs to be reinvented. British Journal of Psychotherapy, 25, 91-109.

Wampold, B. E., \& Imel, Z. E. (2015). The great psychotherapy debate. The evidence for what makes psychotherapy work (2nd ed.). New York: Routledge.

Webb, C. A., DeRubeis, R. J., \& Barber, J. P. (2010). Therapist adherence/competence and treatment outcome: A metaanalytic review. Journal of Consulting and Clinical Psychology, 78, 200-211.

Webb, C. A., DeRubeis, R. J., Dimidjian, S., Hollon, S. D., Amsterdam, J. D., \& Shelton, R. C. (2012). Predictors of patient cognitive therapy skills and symptom change in two randomized clinical trials: The role of therapist adherence and the therapeutic alliance. Journal of Consulting and Clinical Psychology, 80, 373-381.

Weck, F., Grikscheit, F., Höfling, V., \& Stangier, U. (2014). Assessing treatment integrity in cognitive-behavioral therapy: Comparing session segments with entire sessions. Behavior Therapy, 45, 541-552.

Weck, F., Grikscheit, F., Jakob, M., Höfling, V., \& Stangier, U. (2015). Treatment failure in cognitive-behavioural therapy: Therapeutic alliance as a precondition for an adherent and competent implementation of techniques. British Journal of Clinical Psychology, 54, 91-108.

Weck, F., Richtberg, S., Jakob, M., Neng, J. M. B., \& Höfling, V. (2015). Therapist competence and therapeutic alliance are important in the treatment of health anxiety (hypochondriasis). Psychiatry Research, 228, 53-58.

Weck, F., Rudari, V., Hilling, C., Hautzinger, M., Heidenreich, T., Schermelleh-Engel, K., \& Stangier, U. (2013). Relapses in recurrent depression 1 year after maintenance cognitive-behavioral therapy: The role of therapist adherence, competence, and the therapeutic alliance. Psychiatry Research, 210, 140-145.

Westen, D., Novotny, C. M., \& Thompson-Brenner, H. (2004). The empirical status of empirically supported psychotherapies: Assumptions, findings, and reporting in controlled clinical trials. Psychological Bulletin, 130, 631-663.

Wilson, G. T. (1997). Treatment manuals in clinical practice. Behaviour Research and Therapy, 35, 205-210.

Woodward, J. (2003). Making things happen: A theory of causal explanation. Oxford: Oxford University Press.

Wright, J., Sabourin, S., Mondor, J., McDuff, P., \& Mamodhoussen, S. (2007). The clinical representativeness of couple therapy outcome research. Family Process, 46, 301-316.

How to cite this article: Truijens F, Zühlke-Van Hulzen L, Vanheule S. To manualize, or not to manualize: Is that still the question? A systematic review of empirical evidence for manual superiority in psychological treatment. J. Clin. Psychol. 2018;1-15. https://doi.org/10.1002/jclp.22712 